\title{
Determining distances to stars statistically from photometry
}

\author{
Heidi Jo Newberg \\ Rensselaer Polytechnic Institute, Department of Physics, Applied Physics, \& Astronomy, \\ $1108^{\text {th }}$ St., Troy, NY 12180, USA \\ email: newbeh@rpi.edu
}

\begin{abstract}
In determining the distances to stars within the Milky Way galaxy, one often uses photometric or spectroscopic parallaxes. In these methods, the type of each individual star is determined, and the absolute magnitude of that star type is compared with the measured apparent magnitude to determine individual distances. In this paper, we define the term statistical photometric parallax, in which statistical knowledge of the absolute magnitudes of stellar populations is used to determine the underlying density distributions of those stars. This technique has been used to determine the density distribution of the Milky Way's stellar halo and its component tidal streams, using very large samples of stars from the Sloan Digital Sky Survey. Most recently, the volunteer computing platform MilkyWay@home has been used to find the best-fitting model parameters for the density of these halo stars.
\end{abstract}

Keywords. methods: data analysis, methods: statistical, stars: distances, stars: statistics, globular clusters: general, Galaxy: stellar content, Galaxy: structure

\section{Introduction}

We often use the stars in the Milky Way to trace its structure. The brightest stars, which can be used to trace Galactic structure to the largest distances, include blue horizontal branch stars, O, B, and A main-sequence stars, RR Lyraes, Cepheid variables, red clump stars, and $\mathrm{K}$ and $\mathrm{M}$ giants (see Fig. 1). All of these stellar types are reasonably well calibrated as distance indicators. K-giant and red clump stars almost always require spectroscopy to accurately distinguish them from the more numerous main-sequence stars of the same color, and RR Lyrae stars are usually identified from multiple epochs of photometry. All of these bright stars are relatively rare in any stellar population, and many of them are only observed in certain populations. For example, blue horizontal branch stars and RR Lyrae stars are only found in old populations, M giants are only found in relatively metal-rich populations, and $\mathrm{O}, \mathrm{B}$, and $\mathrm{A}$ main-sequence stars are only found in very young populations (although A stars with main-sequence gravities are found in some old populations as blue stragglers).

Deep, large-sky-area, multicolor surveys with high-accuracy calibrations, like the Sloan Digital Sky Survey (SDSs; York et al. 2000) enable new methods of studying the structure of the Milky Way. For example, Ivezić et al. (2008) derived a formula that allows us to estimate the temperature and metallicity of main-sequence stars of type $G$ and later from SDSS photometry. Since these lower-mass main-sequence stars do not evolve over the age of the Universe, their absolute magnitudes are independent of age. Photometry cannot tell us the surface gravities of these types of stars (Lenz et al. 1998), but since the vast majority of the red stars in the photometric survey are main-sequence stars, this is not a major obstacle. Jurić et al. (2009) used this technique to measure the distances to 48 million stars, and then used these distances to determine the density distributions of the disk and halo at $100 \mathrm{pc}$ to $20 \mathrm{kpc}$ from the Sun, over 6500 square degrees of sky. 
Although this analysis used a large number of stars, and resulted in a measurement of stellar density, the Jurić analysis used photometric parallax; they determined the distance to each star individually using photometry.

In this paper, we introduce the concept of statistical photometric parallax. We have used this technique most successfully to study the structure of the Galaxy using turn-off

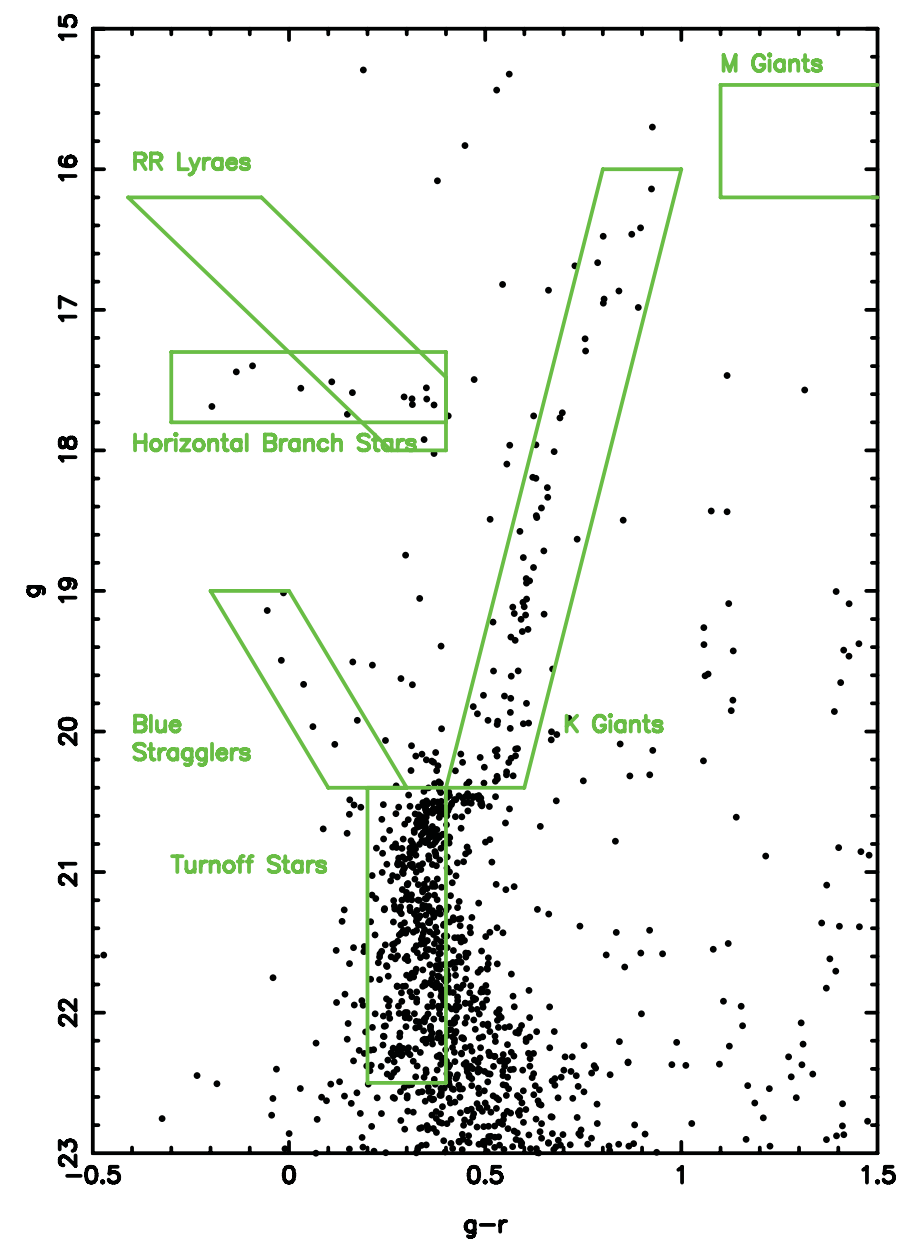

Figure 1. Color-magnitude diagram of stars withing 4 arcminutes of the globular cluster Palomar 5 (Pal 5). All stars within four arcminutes of Pal 5 were selected from sDSS DR9 (SDSS III collaboration 2012). We illustrate how sparse the brighter stars are in a stellar population, compared to the main-sequence and turn-off stars. Most of the selection boxes for each type of star were drawn to include the representative stars in Pal 5. The RR Lyrae selection box was taken from Rave et al. (2003). The M-giant selection box was estimated from Yanny et al.'s (2009) measurements of $M$ giants in the Sagittarius dwarf tidal stream. There are no representatives of these types of stars in the Pal 5 globular cluster. Although giant stars of all types can be observed at larger distances, many types of giant stars are specific to particular populations. $\mathrm{K}$ giants are seen in all but the youngest stellar populations, but these stars are more difficult to separate from dwarf $\mathrm{K}$ stars that have the same colors. Also, K-giant stars are more difficult to use as distance indicators, because their absolute magnitude is a strong function of color (temperature), and is also sensitive to metallicity and age. The drawback to using turn-off stars as distance indicators is that they vary in absolute magnitude by two magnitudes. Statistical photometric parallax allows us to used them effectively to determine the underlying density of stars even though we cannot accurately measure the distance to each individual star. 
stars (Newberg et al. 2002). These stars are by definition brighter than main-sequence stars such as those used by Jurić et al., so they can trace the structure of the Milky Way at $30 \mathrm{kpc}$ or more from the Sun. However, the turn-off stars in a single stellar population can differ in absolute magnitude by two magnitudes (producing a distance error of a factor of 2.5). We do not have a way to determine the distance to a single turn-off star with reasonable accuracy. However, it has been shown that the absolute magnitude distributions of turn-off stars in halo globular clusters are surprisingly similar to each other (Newby et al. 2011), over a metallicity range $-2.3<[\mathrm{Fe} / \mathrm{H}]<-1.2$ dex and ages ranging from 9 to 13.5 Gyr. Recently, Grabowski et al. (2012) showed that this similarity even holds for the globular cluster Whiting 1 , which is thought to be only 6.5 Gyr old (Carraro et al. 2007).

This striking similarity between the absolute magnitude distributions of turn-off stars was not expected. One expects that younger globular clusters would have brighter, bluer turn-off stars. Also, one expects that more metal-rich clusters will have dimmer, redder turn-off stars. As it turns out, older stars in the Milky Way generally have lower metallicity, and the two effects cancel each other. This appears to be an unanticipated consequence of the Milky Way's age-metallicity relationship (AMR; Muratov \& Gnedin 2010; Dotter et al. 2011). Apparently, the absolute magnitude distribution of turn-off stars is similar over the full age and metallicity range of typical stellar populations in the Milky Way halo.

We will describe here the general techique of statistical photometric parallax, which can be used to statistically account for the effects of a range of intrinsic brightnesses of the stellar population which is being used to trace Milky Way's density structure, and can also statistically account for the observational biases in a survey such as the SDSs. We have implemented this technique as a search for the density parameters with the highest likelihood of matching the observed data. Because this parameter search can be computationally expensive, we have employed supercomputers and a large volunteer computing platform, MilkyWay@home, that was built to solve this problem.

\section{Using Statistical Photometric Parallax}

We define the term statistical photometric parallax here for the first time, and it is intended to apply in general to any case where the statistical distribution of absolute magnitudes is used to find the underlying density distribution of stars. However, we will describe here as an example the application of this technique to determine halo substructure using color-selected F turn-off stars, as used by Newberg et al. (2002), Cole et al. (2008), and Newby et al. (2012).

In Newberg et al. $(2002)$, stars with colors $0.1<(g-r)_{0}<0.3 \mathrm{mag}$ and $(u-g)_{0}>0.4$ mag were selected as turn-off stars. The color range was chosen to be bluer than the turnoff of the thick disk, so that halo stars would preferentially be selected. In this paper, only the simplest form of statistical photometric parallax was employed. The distance to the Sagittarius dwarf tidal stream was determined by assuming that the center of the absolute magnitude distribution of turn-off stars in the $g$ filter was $M_{g}=4.2 \mathrm{mag}$. This number was calculated by comparing the apparent magnitude of the turn-off to the apparent magnitude of RR Lyrae stars in the same stellar population. In this example, distances to single stars were not calculated; instead, we made a more accurate determination of distance by looking at the distribution of apparent magnitudes of a particular set of stars.

In Cole et al. (2008), an algorithm was presented that allowed us to determine not just the distance to the center of a stream, but the 3D density of turn-off stars. The 
technique used maximum likelihood to find the model parameters $\overrightarrow{\mathcal{Q}}$ that make the observed star positions $\left(l_{i}, b_{i}, g_{i}\right)$ the most likely. The likelihood $\mathcal{L}$ is given by the product of the probability density functions (PDFs) evaluated at all of the star positions:

$$
\mathcal{L}=\prod \operatorname{PDF}\left(l_{i}, b_{i}, g_{i} \mid \overrightarrow{\mathcal{Q}}\right) .
$$

The PDF is constructed through the following steps:

(a) For each stellar component, one assumes a parameterized model (for example a double exponential, Navarro, Frenk \& White, Hernquist, etc.) for the spatial density.

(b) This spatial density is transformed to $(l, b, g)$ coordinates, assuming that the absolute magnitude of each of the stars is the average for the population.

(c) This density is convolved with the absolute magnitude distribution of the tracers, so that we produce the distribution that we expect to observe.

(d) This expected distribution is multiplied by the completeness for observing stars of a given apparent magnitude in a given survey, as a function of apparent magnitude.

(e) The resulting distribution is normalized so that the integrated probability of finding a star in the entire volume observed is one.

(f) The final PDF is the sum of the fraction of stars in each component times the normalized distribution, summed over the number of components in the model. The fraction of stars in each component are also parameters that are fitted in the maximumlikelihood optimization.

Of these steps, the most time-consuming is the calculation of the integral over the volume. Contrary to first impressions, the time to calculate the likelihood depends more heavily on the number of subvolumes into which the survey space needs to be divided to achieve an accurate result, than on the number of stars in the data set.

One then uses an optimization technique to find the model parameters that produce the highest likelihood. When using a supercomputer, we usually use conjugate-gradient descent. This algorithm is sequential; one evaluates the likelihood and the derivatives with respect to each parameter, chooses a direction, then decides how far in that direction to go before repeating that process.

Newby et al. (2012) applied this technique to all of the data available in SDSS DR7 to find the density of the Sagittarius dwarf tidal stream in the North Galactic Cap, and in three SDSs stripes in the South Galactic Cap. One of the advantages of this probablistic technique is that we are able to extract from the sample of 1.7 million turn-off stars a set of 200,000 stars that have the spatial characteristics of the Sagittarius dwarf tidal stream. This is accomplished by generating a random mumber for each star, and using that random number to place it either in the Sagittarius dwarf tidal-stream catalog, with the probability that a star at that position in the Galaxy is in the Sagittarius dwarf tidal stream, or in the catalog of non-Sagittarius halo stars, with the probability that a star at that position in the Galaxy is not in the Sagittarius dwaf tidal stream. Note that if you wanted to find actual stars in the Sagittarius dwarf tidal stream, say for spectroscopic follow-up, you should use the original catalog of stars and select those with the highest probability of being in the Sagittarius dwarf tidal stream. However, the statistically separated catalog we generated facilitates the study of density substructures in the halo. In particular, we can remove the Sagittarius stream from the original stellar sample, so that we can study the smaller tidal streams, and the density structure of the smooth component of the halo. An example stripe analyzed in Newby et al. (2012) is shown in Fig. 2.

In Cole et al. (2008) and Newby et al. (2012), we modeled the distribution of turnoff star absolute magnitudes as a Gaussian centered at $M_{g}=4.2$ mag with a width of 
$\sigma=0.6 \mathrm{mag}$. Since then, we have recognized that the distribution is asymmetric; there are more stars fainter than the maximum than there are brighter than the maximum. More importantly, we have learned that, owing to larger color errors at fainter magnitudes, the absolute magnitude distribution of color-selected stars is different near the survey limit than it is for the brighter stars. This effect is much larger than we expected it to be. Near the survey limit, the majority of the stars are not turn-off stars, but are fainter main-sequence stars that have scattered into our color selection limits due to large measurement errors. Because we are using a statistical approach, this effect can be included in the analysis by varying the absolute magnitude distribution as a function of apparent magnitude. We plan to include this in future analyses.

\section{Processing time and the MilkyWay@home Volunteer Computing Platform}

We originally tried to implement the search for maximumum likelihood on a single CPU. In a single $2.5^{\circ}$-wide SDSs data stripe, we fit two parameters to a smooth halo with a Hernquist profile, and six parameters per tidal debris stream. If there were three tidal streams in a single stripe, there would be 20 parameters. Evaluating the likelihood for one guess for the model parameters currently takes about 4 hours, with most of the time spent integrating the PDF over the survey volume. To optimize 20 parameters requires some 50 likelihood evaluations per conjugate-gradient descent step and 50 steps per maximum-likelihood evaluation. This totals ten thousand hours per optimization (over a year).

Luckily, the optimization is embarrassingly parallel. It is possible to parallelize the integral, since each integral volume calculation is completely independent of the others. We are able to run this algorithm on a 256 node rack of a Blue Gene/L supercomputer. Parallelizing the integral over 256 nodes cuts the time per likelihood calculation down to under a minute. A conjugate-gradient step can then be accomplished in 47 minutes, and 10 iterations can be accomplished in under 8 hours (which is comfortably shorter than the maximum job size allowed in our queue). In practice, we need to try several conjugate-gradient descents to approximate the best parameters. Once they are known approximately, we run of order ten conjugate-gradient descents, starting near the best values. Including submitting jobs a few at a time and waiting for queue time, this process can take a couple of weeks to validate the results for one SDSS stripe.

Currently our best method for computing the parameters is the volunteer computing platform MilkyWay@home. This project is part of the Berkeley Open Infrastructure for Network Computing (BOINC; Anderson et al. 2005) group of volunteer computing platforms. The first and most famous of these is SETI@home. BOINC offers us a template server and database application, and an infrastructure for volunteers to donate their time to our server. We implemented our own server, including the maximum-likelihood algorithm, a set of optimization routines that will run in a heterogeneous, asynchronous parallel computing environment (Desell et al. 2010b), and a modified server application that sends out 'work units' to the volunteers, collects the results, and validates that the results are not in error (Desell et al. 2010a). One of the surprises in operating a BOINC server is that some of the results sent back from the volunteers are not correct, either because their hardware is malfunctioning, they did not update the software correctly, or they purposely sent back a wrong answer quickly so that they can accumulate BOINC 'credit' more quickly. It is impossible to overestimate how important it is to our volunteers that they get credit for the work units their computers crunch, and that credit is apportioned fairly between the volunteers. 
MilkyWay@home is currently delivering 0.5 PetaFLOPS of computing power from 25,000 active volunteers, giving us access to over 35,000 CPUs or GPUs. The majority of the computing power comes from the GPUs, the best of which can process our likelihood calculations roughly 100 times faster than the CPUs (Desell et al. 2009). It is not easy to parallelize the computation of the integral on BOINC, because that would require communication between the processors, which is not possible at this time. Instead, we parallelize the calculations by sending a single likelihood calculation (including the whole
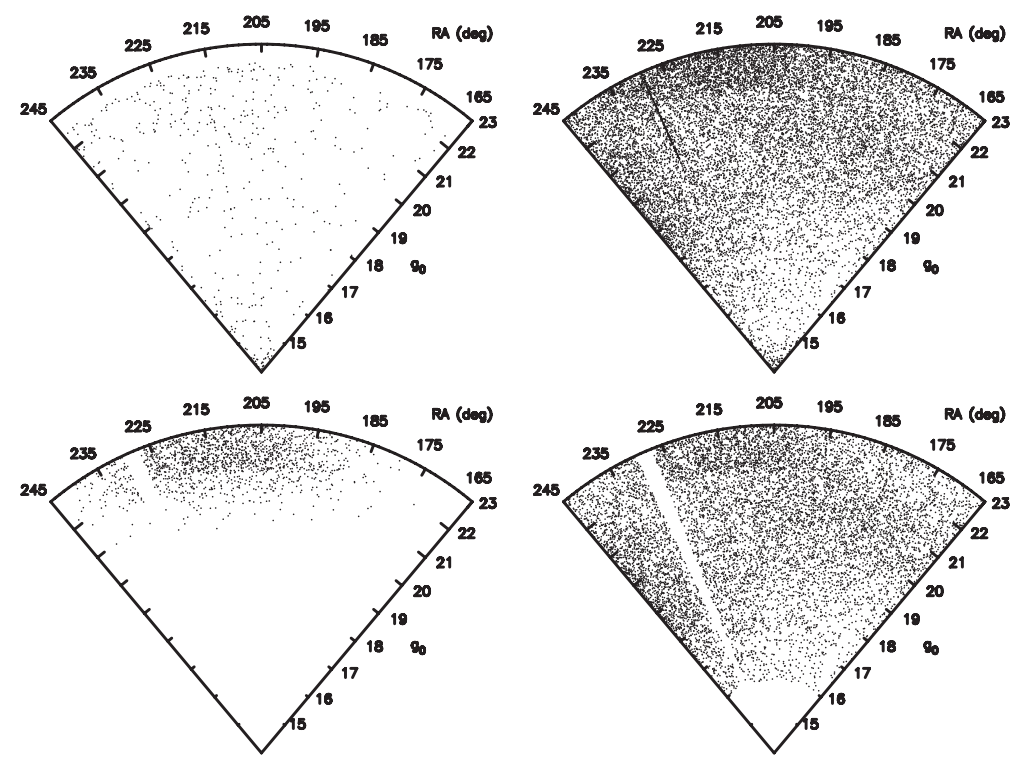

Figure 2. A- and F-star distribution on the Celestial Equator. All stars in this figure were selected within $0.1^{\circ}$ of the Celestial Equator, and at $145^{\circ}<$ R.A. $<265^{\circ}$. The top left-hand panel shows photometrically selected A stars from SDSS DR9 (SDSS III collaboration 2012). The stars were selected with $-0.3<(g-r)_{0}<0.0,0.8<(u-g)_{0}<1.5$, and $14<g_{0}<22.5 \mathrm{mag}$, where the subscript ' 0 ' indicates that the values have been corrected for reddening using the extinction calculated in the database. The top right-hand panel shows photometrically selected turn-off stars, selected from SDSS DR9. The stars were selected with $0.2<(g-r)_{0}<0.4$ and $14<g_{0}<23$ mag. Note that even though we selected only the bluest turn-off stars, there are very many more blue turn-off stars than stars with the colors of A stars. However, the wide range of $g_{0}$ magnitudes observed in the globular cluster $\mathrm{Pal} 5$ at $\mathrm{RA}=229^{\circ}$ indicates that the range of absolute magnitudes of turn-off stars is at least two magnitudes. Even though the range of absolute magnitudes is large, we can see the Pal 5 globular cluster, the Sagittarius dwarf galaxy tidal stream at $\left(\right.$ R.A., $\left.g_{0}\right)=\left(210^{\circ}, 22.5\right)$, the Virgo Overdensity at $\left(\right.$ R.A., $\left.g_{0}\right)=\left(180^{\circ}, 21\right)$, and stars that are presumably part of the smooth spheroid near the Galactic Center at (R.A., $\left.g_{0}\right)=\left(245^{\circ}, 19\right)$. The substructure in A-type stars is not as evident, partially because there are many fewer A-type stars, partially because we have included both blue stragglers and blue horizontal-branch stars, and partially because not all stellar populations have A-type stars. Blue horizontal-branch stars are approximately 1.5 magnitudes brighter than turn-off stars, and blue stragglers are $\sim 3.5$ magnitudes brigher than turn-off stars. A small number of blue stragglers from the Sagittarius dwarf tidal stream are evident at $g_{0}=21 \mathrm{mag}$ in the A-star panel, but Pal 5 blue stragglers are not evident. We do not see blue horizontal-branch stars from either Pal 5 or the Sagittarius dwarf tidal stream in this plot. One can see the advantage of using turn-off stars in identifying the Sagittarius dwarf tidal stream. The bottom two panels show the stars selected by Newby et al. (2012), from this small region of the sky, that have the density distribution of the Sagittarius dwarf tidal stream (left) and the density distribution for everything else (right). The region around the globular cluster Pal 5 was removed from the data before fitting, so that this globular cluster would not affect the results. 
integral for a given $\overrightarrow{\mathcal{Q}}$ ) to each volunteer. These work units can take a couple of minutes (if the work unit is sent to a GPU) or four or more hours (if it goes to a CPU), but it might take minutes, days, or weeks for the likelihood to be returned depending on how much the volunteer is using the computer for her own purposes, and whether she turns it off.

It takes far more computing power to calculate best-fitting parameters on the MilkyWay@home computing system than on a supercomputer. There are four factors responsible for this: (i) We cannot use sequential searches like conjugate-gradient descent. Instead, we use 'particle swarm' or a genetic search algorithm. In the particle-swarm technique, we send out a random set of guesses that span the parameter space. As the likelihood results from the volunteers come back, we send out more work units with guesses that are closer to the higher likelihoods. This search method requires many more steps, but produces more accurate results. (ii) A fraction (approximately 10\%) of the work units, selected at random, are sent out five times so that we can validate that they are correct. If three or more are returned with the same answer, then the result is validated. We also choose to validate the best likelihoods (since those influence our future guesses), and the likelihoods of users that have submitted previous results that did not validate correctly. (iii) Some of the work units that are sent out are never returned. (iv) Because we can, we run the searches for a longer period of time over a wider range of parameter space, and we get better global values for the parameters (Newby et al. 2012). To optimize one stripe takes 1-2 weeks, and hundreds of thousands of likelihood calculations. There are enough volunteers that we can optimize $4-5$ stripes at the same time and still get the results within the same timescale. By putting more jobs on MilkyWay@home at the same time, we decrease the number of work units that are sent out simultaneously. By waiting a little longer to send out more work units, the results of previous searches can be used to make better guesses of the parameters, so adding more jobs increases the computing time at a rate that is less than linear in the number of jobs.

\section{Discussion and Conclusion}

The purpose of this contribution is to define the term statistical photometric parallax, which allows us to determine the density of a population of stars, even if we cannot determine the distance to each individual star in the population. We find the most likely parameters for the density distribution, given that we know the absolute magnitude distribution and the observational constraints of the observed sample of stars. If the population of stars is located all at the same distance (for example in a globular cluster or tidal stream), then statistical photometric parallax can be used to determine the distance to the stellar population. Because the sDss made available a large, well-calibrated sample of stars with multi-color photometry, this technique has recently become feasible.

The example I present is the use of turn-off stars to determine the density distribution of stars in the stellar halo of the Milky Way. By apparent coincidence, the distribution of absolute magnitudes of turn-off stars is very similar for all stellar populations in the age and metallicity range of halo stars. This appears to be a result of the Milky Way's AMR. We are in the process of using this technique to accurately map the density distribution of the entire Milky Way stellar halo.

The drawback to this techique is that to use it one must use a maximum-likelihood algorithm that can in some cases require high-performance parallel computing to get an accurate measurement of the parameters in the density function. In the process of learning to use this method, we created a large volunteer computing platform, MilkyWay@home. 


\section{Acknowledgement}

I would like to thank Matthew Newby and Brian Yanny for helping me obtain the data for the figures in this publication. I also thank Matthew Newby and Jeff Carlin for their help in proofreading. This paper is based upon work supported by the National Science Foundation under grant AST 10-09670. I also would like to thank the MilkyWay@home volunteers for providing us with computing power at no cost, and the Marvin Clan for their support. Funding for SDSS III has been provided by the Alfred P. Sloan Foundation, the participating institutions, the U.S. National Science Foundation, and the U.S. Department of Energy's Office of Science. The SDSS III web site is http://www.sdss3.org/.

\section{References}

Anderson, D. P., Korpela, E., \& Walton, R. 2005, The First Int'l Conf. on e-Science and Grid Technol. (e-Science 2005), p. 196

Carraro, G., Zinn, R., \& Moni Bidin, C. 2007, A\&A, 466, 181

Cole, N., Newberg, H. J., Magdon-Ismail, M., et al. 2008, ApJ, 683, 750

Desell, T., Waters, A., Magdon-Ismail, M., et al. 2009, PPAM, Part I, Lect. Notes Comp. Sci., 6067,276

Desell, T., Magdon-Ismail, M., Szymanski, B., Varela, C., Newberg, H., \& Anderson, D. 2010a, DAIS, Lect. Notes Comp. Sci., 6115, 29

Desell, T., Anderson, D., Magdon-Ismail, M., Szymanski, B., Newberg, H. J., \& Varela, C. 2010b, in: The 2010 IEEE congress on evolutionary computation (IEEE CEC)

Dotter, A., Sarajedini, A., \& Anderson, J. 2011, ApJ, 738, 74

Grabowski, K., Newby, M., \& Newberg, H. J. 2012, J. Undergrad. Res. Phys., submittted

Ivezić, Ž., Sesar, B., Jurić, M., et al. 2008, ApJ, 684, 287

Jurić, M., Ivezić, Ž., Brooks, A., et al. 2008, ApJ, 673, 864

Lenz, D. D., Newberg, J., Rosner, R., Richards, G. T., \& Stoughton, C. 1998, ApJS, 119, 121

Muratov, A. L. \& Gnedin, O. Y. 2010, ApJ, 718, 1266

Newberg, H. J., Yanny, B., Rockosi, C., et al. 2002, ApJ, 569, 245

Newby, M., Newberg, H. J., Simones, J., Cole, N., \& Monaco, M. 2011, ApJ, 743, 187

Newby, M., Cole, N., Newberg, H. J., Desell, T., Magdon-Ismail, M., Szymanski, B., Varela, C., Willett, B., \& Yanny, B. 2012, ApJ, submitted

Rave, H. A., Zhao, C., Newberg, H. J., et al. 2003, ApJS, 145, 245

SDSS III collaboration: Ahn, C. P., Alexandroff, R., Prieto, C. A., et al. 2012, ApJS, submitted (arXiv:1207.7137)

Yanny, B., Newberg, H. J., Johnson, J. A., et al. 2009, ApJ, 700, 1282

York, D. G., Adelman, J., Anderson, J. E., Jr., et al. 2000, AJ, 120, 1579 